\title{
Skin Vesicle
}

National Cancer Institute

\section{Source}

National Cancer Institute. Skin Vesicle. NCI Thesaurus. Code C111969.

A small (less than 5-10 mm) intraepidermal/subepidermal cavity filled with clear, serosang uineous or cloudy fluid. 